\title{
New module integrated multilevel inverter for grid connection of photovoltaic plants
}

\author{
G. Mehlmann ${ }^{1}$, G. Herold ${ }^{1}$ \\ ${ }^{1}$ Institute of Electrical Power Systems \\ University of Erlangen-Nuremberg \\ Cauerstr. 4 - Haus1, 91058 Erlangen (Germany) \\ Phone/Fax number: +0049 913185295 29/41 e-mail: mehlmann@eev.eei.uni-erlangen.de
}

\begin{abstract}
This paper presents a new solar multilevel inverter, based on the cascaded-multilevel converter. The main idea is that the new inverter generates a so called "highly pulsating space phasor" instead of a reference voltage. This operation mode limits the distortions. Additionally, for the first time energy redundancies between the voltage levels can be used, instead of the so far known voltage redundancies. As a result, the problem of unbalanced voltage sources can be avoided, a complex control is not necessary anymore and the MPP tracking of solar multilevel inverters can be improved considerably.
\end{abstract}

\section{Keywords}

Multilevel inverter, pulsating space phasor, energy redundancies, MPP tracking, photovoltaic module

\section{Introduction}

The basic concept of a multilevel converter is to synthesize a staircase voltage waveform by the use of several lower voltage DC sources. A multilevel converter has several advantages, like a high voltage quality and low electromagnetic compatibility problems. Because of the high number of available DC sources one consequential area of application is as interface for photovoltaic plants [1].

Up to now, the staircase voltage waveform of multilevel converters was generally synthesized by multiple DC voltage levels of the same amplitude. But as the duty cycle of each level disagrees, the power flow, associated with each level, is different. Without adequate control techniques this results in unbalanced voltage sources. According to each type of multilevel converter, different strategies, how to balance the voltage sources, were published [1-8]. One of the few works, dealing with differing voltage levels at all is given with [9].

But so far, a concept of generating energy redundancies, by changing the amplitudes of the voltage levels does not exist. In this paper it will be pointed out how to choose the voltage levels, so that energy redundancies appear. These redundancies can be used to achieve equal load- ings of all DC sources and to improve the MPP tracking of solar inverters. Also, a highly pulsating voltage space phasor emerges from the chosen levels. So, the distortions can be decreased much more than in common modulation strategies. As this happens in fundamental frequency modulation the switching losses can be decreased, too. In this connection, a new developed module integrated multilevel inverter for grid connection of photovoltaic plants will be presented.

\section{Pulsating space phasors}

A three-phase system is characterized in each moment by means of three linearly independent time variables $v_{R}(\omega t), v_{S}(\omega t)$ and $v_{T}(\omega t)$. These natural variables allow an adequate specification of the three-phase system.

However, often it is favorably to define a more descriptive mathematical description, like the transformation in space phasor and zero sequence. For any time dependent three-phase system the transformations are

$$
\underline{v}(\omega t)=\frac{2}{3}\left(v_{R}(\omega t)+\underline{a} \cdot v_{S}(\omega t)+\underline{a}^{2} \cdot v_{T}(\omega t)\right)
$$

and

$$
v_{0}(\omega t)=\frac{1}{3}\left(v_{R}(\omega t)+v_{S}(\omega t)+v_{T}(\omega t)\right)
$$

with $\underline{a}=\mathrm{e}^{\mathrm{j} \frac{\pi}{3}}$. This new system is, like the original one, specified by three variables: the zero sequence, the real part of the space phasor and its imaginary part [10].

In a complex coordinate system, the transformation of an ideal sinusoidal symmetrical three-phase system, results in a circular space phasor. A circle is a geometrical figure with infinite axis of symmetry. Hence, it has the highest grade of symmetry. Each divergence of this ideal operation state is directly reflected in the space phasor. Considering a non circular space phasor, the divergence from the ideal space phasor depends on the grade of symmetry. Instead of "grade of symmetry" the term "pulsating" is 
used, as the axis of symmetry can be found in the pulsating structure of the space phasor. With a rising pulse number, respectively a growing grade of symmetry, the three-phase system is getting more and more ideal and the distortions are decreasing.

In general, the occurring harmonics, of a three-phase system, are given against the pulse number [10].

$$
n= \pm p k+1
$$

In equation (3) $n$ is the ordinal number of the occurring harmonics, $p$ is the pulse number and $k \in \mathbb{N}_{0}$.

By means of equation (3) it is quite clearly, that with a rising pulse number, whole groups of harmonics can be eliminated. This is one essential advantage of a highly pulsating operation mode.

Figure 1 shows different pulsating space phasors on the basis of specific time functions. For reasons of clarity, the amplitudes of the space phasors are normalized.

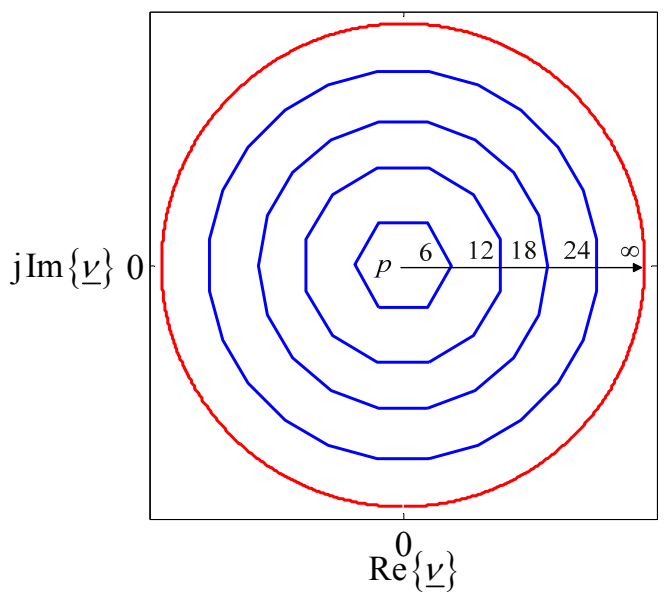

Figure 1: Space phasors of various pulse numbers

Backwards, with the symmetry conditions of a given pulsating space phasor, several corresponding time functions can be found for the phase voltages. One skillful solution for phase $R$ is given with equation (4).

$$
v_{R n}^{\prime}= \begin{cases}\frac{\cos \left(\frac{\pi}{2}-(2 n-1) \frac{\alpha_{p}}{2}\right)}{\cos \left(\frac{\pi}{2}-(2 n-1) \frac{\alpha_{p}}{2}\right)} & \text { for } p=12 i-6 \\ \cos \left(\frac{\alpha_{p}}{2}\right) & \text { for } p=12 i\end{cases}
$$

Here, $\alpha_{p}=2 \pi / p$ is the so called pulse angle, whereas $(n-1) \alpha_{p} \leq \omega t<n \alpha_{p}$ and $n, i \in \mathbb{N}$.

For example, by the use of equation (4), it is now possible to calculate the phase voltage characteristic of a twelve-pulse space phasor. This voltage curve is composed of six constant voltages which are called steps or levels. These levels are given in normalized form in equation (5).

$$
\begin{aligned}
& u_{1}= \pm(2-\sqrt{3}) \\
& u_{2}= \pm(\sqrt{3}-1) \\
& u_{3}= \pm 1
\end{aligned}
$$

Hence, the output voltage of the inverter is defined for a twelve-pulse operation mode. Figure 2 shows the phase voltage of line $R$. The voltage curves of phase $S$ and $T$ are shifted about $2 / 3 \pi$ and $4 / 3 \pi$.



Figure 2: Phase voltage at the inverter output terminal

So, it is possible to define for each pulsating space phasor corresponding time functions. Only the number of levels increases with the pulse-number.

To generate the voltage curve of Figure 2, voltages of several DC sources, like solar modules, fuel cells, batteries or capacitors, must be added. The configurations of these levels and the resulting proportions of the voltage sources are given in equation (6).

$$
\begin{array}{lll}
\left|u_{1}\right|=u_{q 1} & \Leftrightarrow & u_{q 1}=2-\sqrt{3} \\
\left|u_{2}\right|=u_{q 1}+u_{q 2} & \Leftrightarrow & u_{q 2}=2 \sqrt{3}-3 \\
\left|u_{3}\right|=u_{q 1}+u_{q 2}+u_{q 3} & \Leftrightarrow & u_{q 3}=2-\sqrt{3}
\end{array}
$$

Here, it has to be taken in mind that these voltage sources differ in their amplitudes.

By addition of a proper zero sequence it is possible to reduce the number of levels, as pointed out in Figure 3.

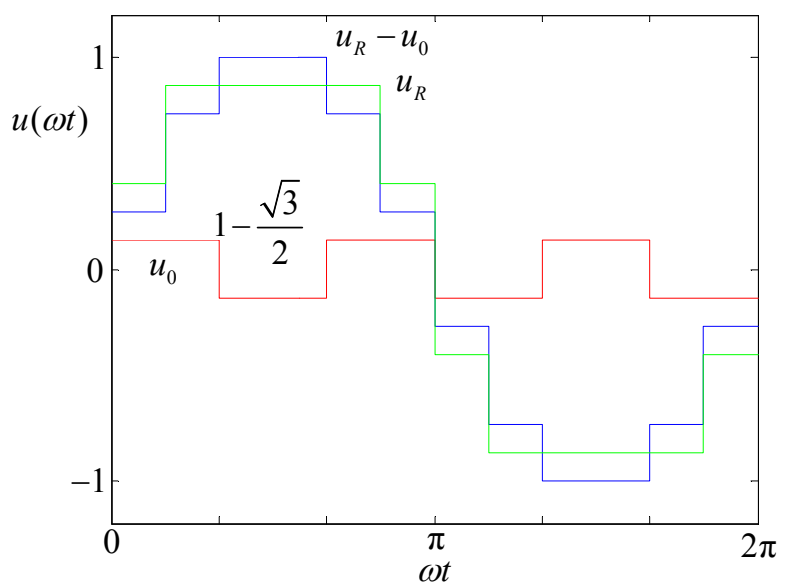

Figure 3: Phase voltage by addition of a zero sequence

For a steady operation mode of the inverter, it is important that the provided energy of each DC source is identical.

Conventional multilevel converters use voltage redundancies and complex modulation strategies to fulfill this condition [1-8]. An inverter with a pulsating space phasor has no voltage redundancies because of the different levels. In fact, each DC source has to generate one associated level for a specified time. But because of the specific chosen voltage levels energy redundancies appear. These redundancies can be used by a proper layout of the 
inverter. For the twelve pulse inverter with zero sequence the energy balance is given with (7).

$$
E_{q 1}=E_{q 2}
$$

Equation (8) specifies the energy balance for a twelve pulse inverter without zero sequence as well as for an 18pulse inverter with zero sequence.

$$
E_{q 2}+E_{q 3}=2 E_{q 1}
$$

Similar redundancies can be found for any pulse numbers.

So, generating pulsating space phasors, energy redundancies can be used. As a result, it is easy to balance all voltage sources and to realize an adequate MPP tracking. The energy redundancies are an extra benefit of this operation mode beside the elimination of harmonics. Furthermore, the phase voltages of a pulsating space phasor can be generated in fundamental frequency modulation.

\section{Solar multilevel inverter}

\section{A. Circuit design and field of application}

In this chapter the circuit design of the multilevel inverter as a module integrated inverter for grid connection of photovoltaic plants will be shown.

Figure 4 shows the schematic of such a six level inverter.



Figure 4: Schematic of a twelve-pulse (18-pulse) solar inverter
The layout bases on the cascaded-multilevel converter. In principle, space phasors with an unlimited pulse number can be generated with this topology. For reasons of clarity, an inverter with the pulse number of twelve or 18 , depending whether a zero sequence is added or not, will be illustrated.

Three solar modules of the same MPP voltage $U_{1}, U_{2}$ and $U_{3}$ and the same peak power are used as power supply. $\mathrm{DC} / \mathrm{DC}$ converters with galvanic isolation are used to generate three voltages per phase: $U_{q 1}, U_{q 2}$ and $U_{q 3}$. These are the three different sources of the pulsating space vector.

The upper DC/DC converter is a simple flyback converter. The lower one connects two sources energy redundant with the output and puts so equation (8) into practice. The voltages $U_{q 1}, U_{q 2}$ and $U_{q 3}$ will automatically appear with the defined amplitude, as long as the feed-in of the sources $U_{1}, U_{2}$ and $U_{3}$ are the same. The reason therefore is the law of energy redundancies. So, it is not necessary to use the DC/DC converters to adjust the amplitudes. This has, for example, the advantage that the DC/DC converters can be used for the MPP tracking of solar modules.

The output terminals of the DC/DC converters are connected with H-Bridges, which work in fundamental frequency modulation. They are controlled in such way, that a highly pulsating space phasor emerges, which is supplying a three-phase power system. With every available DC source the circuit can be enlarged and the pulse number increases. By the use of an adequate zero sequence the pulse number increases by six with every source.

The DC/DC converters are necessary for an adequate MPP tracking [11]. Here, the galvanic isolation is not only necessary because of the circuit layout. If namely a grounded person touches a module without galvanic isolation high shock currents can appear because of capacitive heat losses [12], [13].

With the inverter it is possible to connect solar modules separately with the grid. So each module can work in its MPP. In comparison, the common string concept has just one working point for all modules connected by the string. This working point is of course worse than the MPPs of the individual modules. So with the separate connection of the modules it is possible to increase the feed-in and as a result the salary. Also, a simple and flexible plant layout is possible and it is easy to enlarge the plant later on. So, a convenient and low priced start up with small-sized photovoltaic plants is possible. Furthermore the implementation of diagnostic systems is easy. In the long term, the fabrication of standardized devices in high numbers is possible. The low variability of the module voltages enables a convenient layout of the power electronics [13], [14].

Another advantage is the safeguarding against failure. If for example one module or DC/DC converter fails, it is possible to change the operation mode in such way that the pulse number would decrease by six. Meaning, an 18pulse inverter would work as a twelve pulse inverter.

Because of the multilevel topology multiple modules generate the output voltage curve. So, compared with the conventional methods of grid connection, the electronic devices can be dimensioned smaller. Already with a few 
modules, respectively DC sources, the high pulse operation mode limits the number of harmonics. As the inverter operates in fundamental frequency modulation switching losses can be reduced.

\section{B. MPP tracking}

The energy redundant converter ensures that the feed-in of all sources is equal and that, at the same time, the necessary voltage levels, for a designated pulsating space phasor, result from the charging of the output capacitors. Table 1 gives a review about some redundancies.

Table 1: Energy Redundancies

\begin{tabular}{|c|c|c|}
\hline$p$ & Redundancies with $u_{0}$ & Redundancies without $u_{0}$ \\
\hline 12 & $E_{2}+E_{2}=2 E_{1}$ & $E_{2}+E_{3}=2 E_{1}$ \\
\hline 18 & $E_{2}+E_{3}=2 E_{1}$ & $E_{2}+E_{5}=E_{3}+E_{4}=2 E_{1}$ \\
\hline 24 & $E_{1}+E_{3}=E_{2}+E_{4}=2 E_{1}$ & $E_{1}+E_{4}=E_{2}+E_{6}=E_{3}+E_{5}=2 E_{1}$ \\
\hline 30 & $E_{2}+E_{5}=E_{3}+E_{4}=2 E_{1}$ & $E_{1}+E_{5}=E_{2}+E_{8}=E_{3}+E_{7}=E_{4}+E_{6}=2 E_{1}$ \\
\hline$\ldots$ & $\ldots$ & $\ldots$ \\
\hline
\end{tabular}

It can be deduced that the collective energy output of two DC sources is always twice as high as the output of the source that generates the first level. For an 18-pulse space phasor with zero sequence as well as for a twelve-pulse space phasor without zero sequence equation (9) is given. $E_{2}+E_{3}=2 E_{1}$

Figure 4 illustrates the technical activation of equation (12). The sources 2 and 3 are connected alternating with the transducers. As a result, the average of the feed-in is for both sources $E_{1}$ and the energies at the transducers are always $E_{2}$ and $E_{3}$. Here the sources are connected with the transducers by two H-bridges. On the analogy of the Flyback converter, the semiconductor switches of each bridge operate with a high frequency and control the power flow. The working point setting of the converter takes place during two switching cycles. With

$\frac{1}{2}\left(E_{2}+E_{3}\right)=E_{1}=E_{\mathrm{MPP}}$

it is guaranteed that the modules operate in the Maximum Power Point. At the same time, because of

$\frac{E_{2}}{E_{3}}=k$

the ratio of energies is consistent with the given ratio $k$ for a designated pulsating space phasor.

Whereas equation (11) is fixed, $E_{\mathrm{MPP}}$ in (10) depends among other things on the radiation and cell temperature. By solving this system of equations the energies and consequently the duty cycles of the semiconductor switches are given.

$$
\begin{aligned}
& E_{2}=\frac{2 E_{1}}{1+\frac{1}{k}}=\frac{2 E_{\mathrm{MPP}}}{1+\frac{1}{k}} \\
& E_{3}=\frac{2 E_{1}}{1+k}=\frac{2 E_{\mathrm{MPP}}}{1+k}
\end{aligned}
$$

The associated switching states of the semiconductors as well as the currents are illustrated in Figure 5.

So, it is possible that the output capacitors in Figure 4 are charged with the energies $E_{1}, E_{2}, E_{3}$ whereas the feed-in of all DC sources is $E_{1}$. As result a pulsating space phasor occurs and all DC sources and capacitor voltages are balanced.

With this modified DC/DC converter it is possible to generate space phasors with high pulse numbers and to realize an adequate MPP tracking. Instead of solar modules other DC sources can be controlled and balanced with this procedure, too.



Figure 5: Switching states of the energy redundant DC/DC converter

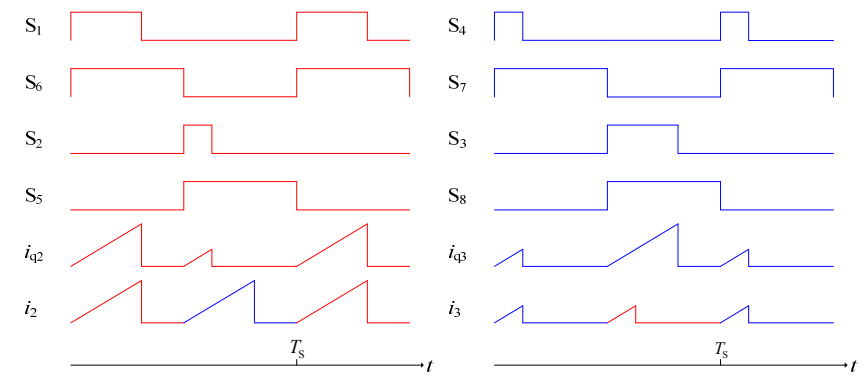

Figure 6: Switching states and input currents

In general, two techniques of MPP tracking are possible. The first one is the yield optimized MPP tracking. Here, all modules are controlled independently and each one is working in its MPP. So, the maximum feed-in of the plant is given. But, deviations of the feed-in, for example because of partial shadowing, can result in an unsymmetrical space phasor.

Alternative, the grid optimized MPP tracking ensures a symmetrical operation mode. For this purpose, the module with the lowest feed-in is working as master and the others as slaves. The power output of the master is the nominal power of all slaves. As a result the distortions are always minimized, but the feed-in is lower.

Alike a combination of both MPP-tracking methods is possible, too. So, if the distortions reach a critical value, it would be possible to change the yield optimized MPP tracking in the grid optimized.

In general the yield optimized MPP tracking is preferable, as the distortions in a highly pulsating operation mode are very low and this is even so if several modules totally fail.

\section{Results}

Already, with a few modules it is possible to generate voltage- and current curves that are nearly free of harmonics. For example with eight modules it is possible to generate a space phasor with the pulse number 48 and total voltage distortions of only $3,8 \%$, whereas all numbers of harmonics $n$ were taken into consideration $\left(T H D_{n \rightarrow \infty}\right)$. General, inverters with a pulse number above 
48 are expedient. Practical, no distortions exist in the voltage and current forms of such an inverter. By definition the $T H D$ for such pulse numbers is zero, as only the first 40 numbers of harmonics have to be taken into consideration [15]. And these harmonics do not exist in a space phasor with a pulse number above 42 .

Following some results for a twelve-pulse inverter, supplied by two photovoltaic modules, are shown. This inverter is the smallest possible implementation and most suitable to demonstrate the highly pulsating operation mode.

Figure 5 illustrates the distortions of the output current for different load ratios and a varying power input. Along the dotted line, where the feed-in of both modules is identical, is the defined operation mode of the inverter. The distortions are minimal in this operation mode. Beyond this line the pulse number of the inverter is six, whereas an approximated twelve-pulse operation mode is given nearby the line. So, small fluctuations of the feedin have nearly no influence.

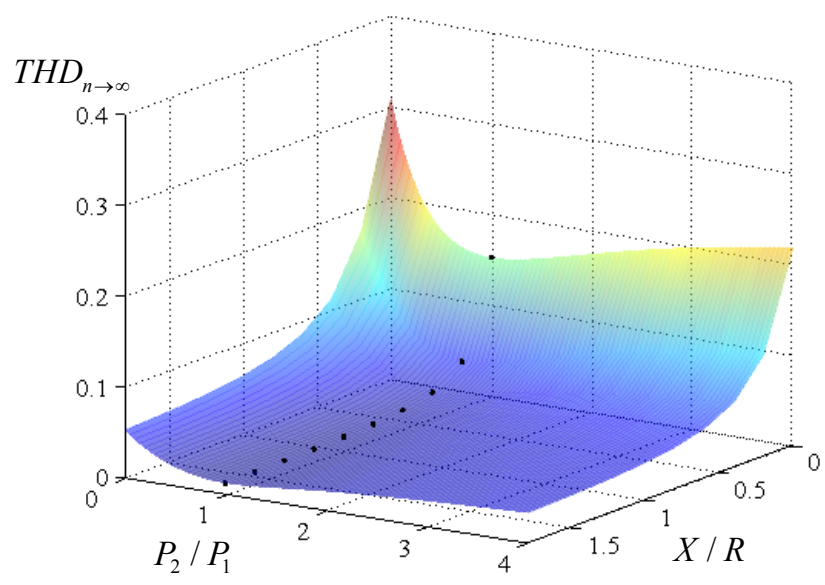

Figure 7: Distortions by variation of the feed-in and load ratio

Figure 6 shows the dedicated characteristics of the seventh voltage harmonic. Along the line of the twelve pulse operation mode it is zero. Beyond this working line it increases up to $14,3 \%$. At this point the space phasor has an ideal six pulse character and the impedance is zero.



Figure 8: Seventh harmonic by variation of the feed-in and load ratio
At last, Figure 7 demonstrates the course of the voltage space phasor by variation of the feed-in. If only one photovoltaic module is working, the space phasor has the pulse number of six. Here the voltage phasor jumps from corner to corner and remains there for the time of

$t=\frac{2 \pi}{p}$

with $p=6$. With increasing feed-in of the second module the space phasor passes into a space phasor with the pulse number of twelve. For $P_{1}=P_{2}$, the space phasor reaches the twelve pulse operation mode. Now the space phasor remains in each corner for the time defined in (16), whereas $p=12$. The distance between the corners is exactly of the same length.

With increasing feed-in of the second module the space phasor passes into a space phasor with the pulse number of twelve. For $P_{1}=P_{2}$, the space phasor reaches the twelve pulse operation mode. For continuous increasing feed-in of the second module the space phasor has a six-pulse character again.

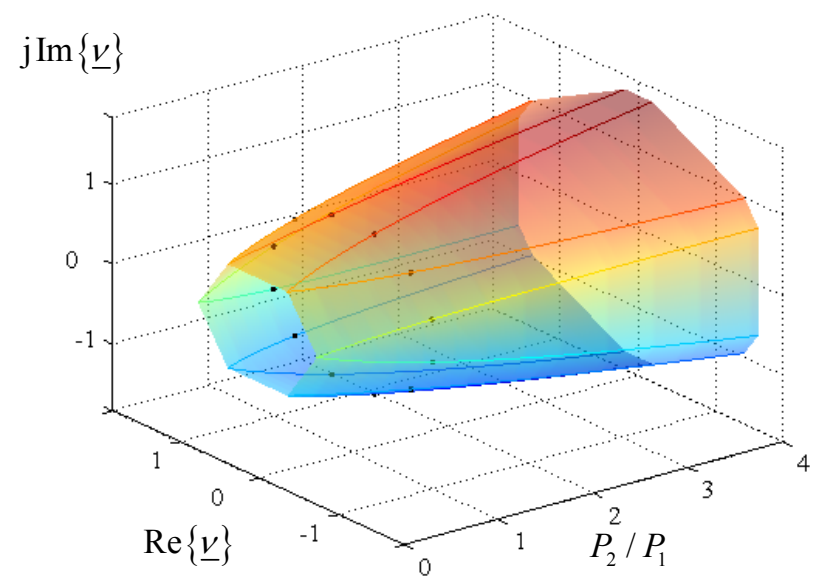

Figure 9: Space phasor by variation of the feed-in

\section{Conclusion}

In this paper a way was illustrated, how to generate AC voltages with low distortions in fundamental frequency modulation. Here, the idea of pattering a reference voltage was left behind. Instead, directly a highly pulsating space phasor was generated.

Also, a so far unknown correlation between the energies, belonging to special chosen voltage levels of a space phasor, was presented. Because of this correlation, energy redundancies can be used to balance the voltages of a multilevel inverter and to improve the MPP tracking of solar multilevel inverters.

A circuit to implement these theoretical concepts was illustrated and explained.

At last the application area as photovoltaic inverter was discussed and results of the circuit in different operating conditions were shown.

The presented multilevel inverter is just one technical possibility to generate highly pulsating space phasors. In principle, the described methods are applicable for other multilevel converters, too. 


\section{References}

[1] S. Khomfoi and L. M. Tolbert, Power Electronics Handbook, 2. Edition, Chapter 31 - Multilevel Power Converters, Elsevier, Amsterdam (2007)

[2] L. M. Tolbert and F. Z. Peng, "Multilevel Converters as a Utility Interface for Renewable Energy Systems", in Proc. IEEE/PES Summer Meeting 2000, Vol. 2, pp. 1271-1274

[3] L. M. Tolbert, F. Z. Peng, T. Cunnyngham and J. N. Chiasson, "Charge Balance Control Schemes for Cascade Multilevel Converter in Hybrid Electric Vehicles", IEEE Transactions on Industrial Electronics 2002, Vol. 49, No. 5, pp. $1058-1064$

[4] K. A. Corzine and X. Kou, "Capacitor Voltage Balancing in Full Binary Combination Schema Flying Capacitor Multilevel Inverters", IEEE Power Electronic Letters 2003, Vol. 1, No. 1

[5] M. Marchesoni and P. Tenca: "Diode-Clamped Multilevel Converters: A Practiable Way to Balance DC-Link Voltages", IEEE Transactions on Industry Applications 2002, Vol. 49, No. 4, pp. 752-765

[6] M. Marchesoni, M. Mazzucchelli and P. Tenca, "About the DC-link capacitors voltage balance in multi-point clamped converters", in Proc. IEEE IECON1998,

[7] A. Lesnicar and R. Marquardt, "A new modular voltage source inverter topology", in Proc. EPE2003

[8] A. Antonopoulos, L. Ängquist and H. P. Nee, "On Dynamics and Voltage Control of the Modular Multilevel Converter", in Proc. EPE2009

[9] A. J. Iwaszkiewicz and B. J. Perz, "A Novel Approach to Control of Multilevel Converter Using Wavelets Transform", in Proc. ICREPQ2007

[10] G. Herold, Elektrische Energieversorgung I, Drehstromsysteme - Leistungen - Wirtschaftlichkeit, J. Schlembachverlag Weil der Stadt (2002)

[11] T. Esram and P. L. Chapman, "Comparison of Photovoltaic Array Maximum Power Point Tracking Techniques", IEEE Transactions on Energy Conversion 2007, Vol. 22, No. 2

[12] J. C. Hernández and P. G. Vidal, "Guidelines for Protection Against Electric Shock in PV Generators", IEEE Transactions on Energy Conversion 2009, Vol. 24, No. 1

[13] J. M. A. Myrzik and M. Calais, "String and Module Integrated Inverters for Single-Phase Grid Connected Photovoltaic Systems - A Review", in Proc. IEEE PTC2003

[14] M. Viotto, "Photovoltaik - Neue Horizonte, Solarmodule mit integriertem Wechselrichter", Jahrestagung des ForschungsVerbunds Sonnenenergie 2003, pp. 71-75

[15] G. Herold, Elektrische Energieversorgung V, Stromrichter in Drehstromnetzen, J. Schlembachverlag, Wilburgstetten (2009) 